Ann. Parasitol. Hum. Comp., 1992, $67: \mathrm{n}^{\circ} 6,180-187$.

Mémoire.
Key-words: Protostrongylidae. Intermediate host. Cochlicella barbara (Mollusca). Natural infection. Periods of risk. Definitive hosts.

Mots-clés : Protostrongylidae. Hôte intermédiaire. Cochlicella barbara (Mollusca). Infestation naturelle. Périodes à risque. Hôtes définitifs.

\title{
NATURAL INFECTION OF COCHLICELLA BARBARA (MOLLUSCA) BY PROTOSTRONGYLIDAE (NEMATODA) IN PADDOCKS WITH DIFFERENT GRASS HEIGHTS IN THE NORTHWEST OF SPAIN: PERIODS OF RISK FOR THE DEFINITIVE HOSTS
}

\author{
P. MORRONDO-PELAYO*, P. DIEZ-BAÑOS*, M. MEZO-MENENDEZ**, \\ N. DIEZ-BAÑOS ***, G. FLORES-CALVETE **
}

\begin{abstract}
SUMMARY
Throughout a year a study was made of the number of adult and juvenile Cochlicella barbara present in four paddocks with similar edapho-topographic and botanic characteristics, in which four grass heights were established. The sheep were naturally infected by bronchopulmonary nematodes when grazing, on the permanent pasture. Adult molluscs were found in a greater number than juvenile ones and they presented a greater prevalence and intensity of infection.
\end{abstract}

The different climatic factors considered had a greater influence on the infection of juvenile snails than on adults. In both cases the temperature presented a positive correlation with the $C$. barbara infection intensity.

The sheep were more likely to get infected from mid spring to mid autumn, and particularly in summer (all the paddocks remained infected during the grazing season, and the paddock with the lowest height of grass was more infected than the others).

Résumé : Infestation naturelle de Cochlicella barbara (Mollusca) par des Protostrongylidae (Nematoda) en parcelles avec différentes hauteurs d'herbe dans le nord-ouest de l'Espagne : périodes à risque d'infestation pour les hôtes définitifs.

La population de Cochlicella barbara (adulte et juvénile) de quatre parcelles a été étudiée pendant un an. Les caractéristiques pédologiques, topographiques et botaniques sont similaires pour ces parcelles, dans lesquelles on a établi quatre hauteurs d'herbe. Des moutons infestés naturellement par des nématodes bronchopulmonaires utilisent ce pâturage en permanence. Les mollusques adultes ont été trouvés en plus grand nombre que les mollusques juvéniles et ils ont présenté une fréquence et une intensité d'infestation supérieures. Les différents facteurs climatiques considérés ont influé davantage sur les juvéniles que sur les adultes. La température est dans les deux cas celle qui a présenté la plus grande corrélation positive avec le degré d'infestation de Cochlicella barbara.

Les ovins peuvent s'infester du printemps à l'automne, et surtout en été, vu que dans les différentes parcelles, et spécialement dans celles de plus petite hauteur d'herbe, il y a eu de nombreux mollusques avec des larves infestantes disponibles pour les hôtes définitifs.

\section{INTRODUCTION}

Protostrongylidae are pulmonary parasites of small ruminants and have a complex life cycle that begins with the elimination of first-stage larvae (L1) in the faeces from definitive hosts. Several environmental factors influence the survival of $\mathrm{L} 1$. It is necessary that these $\mathrm{L} 1$ penetrate a mollusc that acts as an intermediate host adequate for them to develop until third-stade larvae (L3), which, in their

* Departamento Patología Animal (Parasitología y Enfermedades parasitarias), Fac. Veterinaria Lugo, Univ. Santiago de Compostela (Spain).

** Centro de Investigaciones Agrarias, Mabegondo (La Coruña, Spain).

*** Departamento Patología Animal (Sanidad Animal), Fac. Veterinaria, Univ. León (Spain).

Accepté le : 24 mars 1992. turn, are the infective stages for sheep and goats. Climatic conditions have a remarkable influence on the infection rate of molluscs and on the subsequent larval development (Cabaret, 1988).

The control of these pulmonary parasitisms is not only achieved by the treatment of the ultimate hosts with anthelmintics, since, if the infected molluscs remain in the grazing lands, the cycle may continue. The prophylactic measures in these parasitisms must include the knowledge of the infection rate and number of molluscs present in the different seasons of the year and in the different sorts of grazing lands so as to be able to determine which periods imply a greater risk for the infection of the small ruminants.

The aim of this work is to contribute to the clarifying of the influence of the climatic factors over the external cycle of the Protostrongylidae in a humid area of Northwest of Spain. During a year, the larval elimination rythms 
were studied in a flock of sheep in permanent grazing divided, into 4 types of paddocks with different grass heights. In these a great population of Cochlicella barbara, a terrestrial gastropod mollusc, was found. This mollusc acts, as stated in the revision carried out by Manga et al. (1986), as an adequate intermediate host for Protostrongylidae larvae, both in experimental and natural infections.

\section{MATERIAL AND METHODS}

The molluscs were collected weekly, between December 1988 and November 1989 in an estate in the Centre for Agricultural Research in Mabegondo (La Coruña, Spain). The work was carried out in an area of $25,000 \mathrm{~m}^{2}$.

The structure of the soil is of the slime-sandy type, with a $\mathrm{pH}$ of 6.1. The sheep were kept in paddocks seeded with graminae (Lolium perenne and Dactilys glomeratus) and leguminous plants (Trifolium repens). The meteorological data were obtained in the observatory of the centre where the study was carried out. The average of the minimum, maximum and average temperatures was considered monthly, as well as the average relative humidity, average precipitation and days of frost, rain and mist.

The area of study was divided into four neighbouring paddocks of equal size $\left(6,225 \mathrm{~m}^{2}\right.$ each). Four grass heights were trimmed: $3,6,9$ and $12 \mathrm{~cm}$ for paddocks A, B, C and D respectively. These heights were obtained by modifying the stocking rate which fluc- tuated between 6 sheep for the paddock D and 11 sheep per paddock A. When necessary the ovine diet was supplemented with commercial feed in the paddocks.

The sheep were of Galician breed and their age ranged between 1 and 8 years,. Monthly faecal larval counts were carried out. The prevalence and highest intensity corresponded to Neostrongylus linearis and in a lower proportion to Cystocaulus ocreatus and Muellerius capillaris.

The collection of snails were made every month as follows: paddocks $A$ and $C$ ( 3 and $9 \mathrm{~cm}$ of grass height) on the first week, $B$ and $D(6$ and $12 \mathrm{~cm})$ on the second, $A$ and $C$ on the third and B and D on the fourth week. In this way, fortnightly data were obtained from each paddock. Throughout the work the same person went across each paddock diagonally (the two diagonals, i.e. $113 \mathrm{~m} \times 2$ in half an hour) and collected the molluscs found (alive or dead).

As the results for each month were similar for every paddock and in order to facilitate the comprehension and statistical treatment, the data are expressed monthly.

The molluscs were killed by immersion for 24 hours in a container with tepid water, after which the species and the age of the molluscs were determined and the number of living and dead ones was quantified.

In the area of study only $C$. barbara was found. Nevertheless in autumn and spring slugs were also found, although in a low number ( 6 as the maximum).

The foot of the molluscs, a preferential area for Protostrongylidae larvae implantation, was examined under the microscope by using the compresorium. The larvae were counted, extracted by

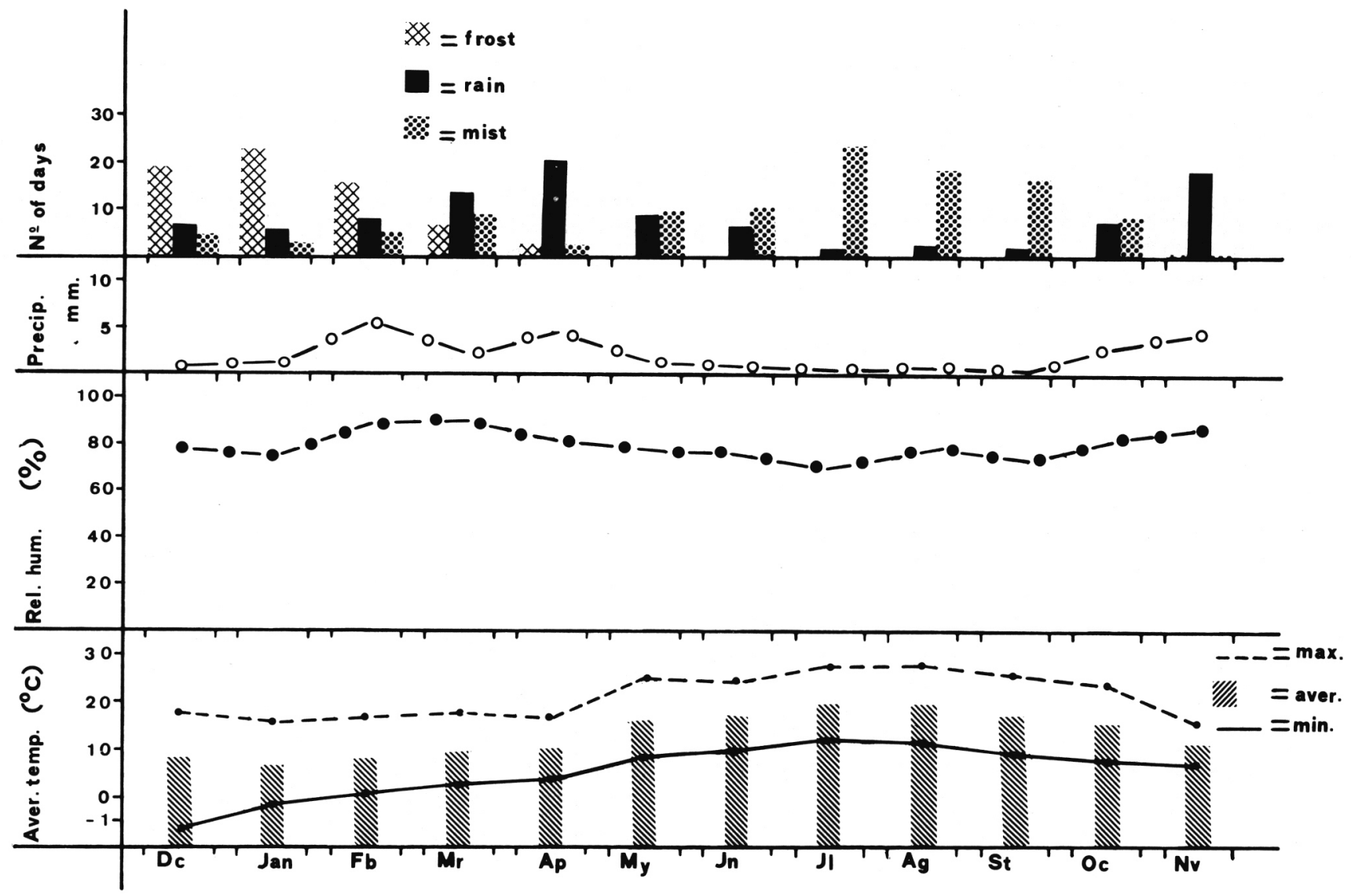

FIG. 1. - Meteorological data in Mabegondo (La Coruña, Spain), from December 1988 to November 1989. 
dissection of the foot of the molluscs using entomological needles and identified.

The Chi-squared test $\left(\chi^{2}\right)$ was used to check whether there were statistically significant differences between adult and juvenile specimens in relation to the number of living, dead or infected molluscs; and in relation to the total number of larvae and L3 found in the snails.

Linear regression was used to check whether there was a correlation between living molluscs, parasitised ones, the total number of larvae and L3 they had and the different climatic parameters (average of the minimum, maximum and average temperatures), rainfall in $\mathrm{mm}$, percentage of relative humidity, number of days of rain, mist and frost.

All calculations were done using the Statgraphics programme (Statistical Graphics Corporation, EXE (1986), version 2.1 and the Stat-Itcf computer package (1988).

\section{RESULTS}

\section{1 - Molluscs population}

In Figure 1 several meteorological data registered from December 1988 to November 1989 are indicated. It was observed that from May to September the temperatures were high and rainfall was scarce. On the other hand, temperatures were very low (mainly the minimum temperatures) and there were many days of frost between December and April. Likewise it was noticed that, particularly in summer, there were many days with morning mist that helped to maintain the environmental relative humidity.

The number of living (Table I) and dead (Table II) molluscs collected during this study is summarized. A total of 6,103 living specimens (3,932 adult and 2,171 juvenile) were found. In every paddock there was a larger number of adult than juvenile specimens, varying greatly depending on the month.

The maximum number of adults was found from August to November for the four grass heights. From December onwards the number decreased progressively, there were

TABLEAU I. - Number of molluscs collected alive in paddocks $A, B, C$ and $D$.

\begin{tabular}{||c||c|c|c|c||c|c|c|c||}
\hline \multirow{2}{*}{ MONTHS } & \multicolumn{3}{|c||}{ ADULT MOLLUSCS } & \multicolumn{3}{|c||}{ JUVENILE MOLLUSCS } \\
\cline { 2 - 10 } & A & B & C & D & A & B & C & D \\
\hline \hline DC & 46 & 45 & 48 & 55 & 2 & 2 & 4 & 5 \\
\hline Jan & 22 & 30 & 7 & 12 & 4 & 2 & 0 & 1 \\
\hline Fb & 41 & 0 & 0 & 14 & 2 & 0 & 2 & 0 \\
\hline Mr & 15 & 10 & 5 & 11 & 3 & 0 & 0 & 0 \\
\hline Ap & 0 & 2 & 0 & 0 & 4 & 11 & 11 & 3 \\
\hline My & 3 & 0 & 3 & 2 & 44 & 88 & 76 & 47 \\
\hline Jn & 0 & 0 & 1 & 1 & 24 & 98 & 89 & 62 \\
\hline Jl & 0 & 0 & 0 & 0 & 136 & 215 & 204 & 120 \\
\hline Ag & 50 & 102 & 60 & 71 & 81 & 173 & 247 & 224 \\
\hline St & 272 & 208 & 233 & 390 & 56 & 56 & 29 & 17 \\
\hline Oc & 200 & 246 & 249 & 359 & 0 & 15 & 3 & 0 \\
\hline Nv & 357 & 276 & 238 & 248 & 3 & 2 & 2 & 4 \\
\hline \hline
\end{tabular}

TABlEaU II. - Number of dead molluscs collected in paddocks $A, B, C$ and $D$.

\begin{tabular}{||c||c|c|c|c||c|c|c|c||}
\hline \multicolumn{1}{|c||}{ MONTHS } & \multicolumn{3}{c||}{ ADULT MOLLUSCS } & \multicolumn{3}{|c||}{ JUVENILE MOLLUSCS } \\
\hline \hline DC & 7 & B & C & D & A & B & C & D \\
\hline Jan & 59 & 25 & 58 & 39 & 9 & 3 & 8 & 5 \\
\hline Fb & 66 & 23 & 22 & 21 & 6 & 0 & 1 & 2 \\
\hline Mr & 11 & 23 & 18 & 22 & 2 & 2 & 2 & 5 \\
\hline Ap & 9 & 5 & 0 & 0 & 0 & 4 & 0 & 3 \\
\hline My & 8 & 3 & 7 & 1 & 8 & 13 & 5 & 0 \\
\hline Jn & 122 & 0 & 2 & 1 & 18 & 21 & 7 & 16 \\
\hline Jl & 9 & 4 & 0 & 0 & 10 & 30 & 99 & 5 \\
\hline Ag & 0 & 0 & 0 & 15 & 22 & 9 & 21 & 11 \\
\hline St & 10 & 28 & 6 & 8 & 0 & 0 & 0 & 0 \\
\hline Oc & 80 & 9 & 6 & 20 & 0 & 8 & 32 & 2 \\
\hline Nv & 7 & 0 & 23 & 5 & 0 & 5 & 0 & 5 \\
\hline
\end{tabular}

even paddocks in which, from February to July, no living specimen was collected. The number of adults collected throughout the work was similar for the four grass heights, with slight variations in favour of the paddock with highest grass.

The juvenile specimens increased from May; in every paddock the highest figures were found in July and August, along with the highest temperatures. During these two months, the rainfall was not very important, although the relative humidity remained high, probably due to the frequent mists registered. From September the number of juvenile specimens decreased and during autumn and winter they were very scarce.

The total number of dead collected specimens (Table II) was 1,108 (697 adult and 411 juvenile). In the colder months (January-March) the highest adult mortality rate was registered, varying in the four paddocks, having a higher value in those with a lower height. The mortality rate among the juvenile specimens was particularly high from June to September (along with the highest figures of living juvenile specimens). From November onwards both living and dead specimens found were very scarce. In the paddocks with the highest grass height fewer juvenile specimens were found dead.

\section{2 - Prevalence and intensity of molluscs infections}

With regard to the parasitised molluscs (Table III), monthly variations were observed in adults, as well as in juvenile specimens. The maximum number of infected molluscs coincided with the periods with a larger number of snails, that is from August to December for adults and from May to September for juvenile specimens. In the paddock with the highest grass (D), the lowest number of adult and juvenile infected specimens was obtained but, however, the highest percentage of parasitised was seen in the paddock with the lowest grass height. We must also point out 
TABLEAU III. - Number of infected molluscs with Protostrongylidae larvae.

\begin{tabular}{||c||c|c|c|c||c|c|c|c||}
\hline \multirow{2}{*}{ MONTHS } & \multicolumn{4}{|c||}{ ADULT MOLLUSCS } & \multicolumn{3}{|c||}{ JUVENILE MOLLUSCS } \\
\cline { 2 - 10 } & $\mathrm{A}$ & $\mathrm{B}$ & $\mathrm{C}$ & $\mathrm{D}$ & $\mathrm{A}$ & $\mathrm{B}$ & $\mathrm{C}$ & $\mathrm{D}$ \\
\hline \hline $\mathrm{DC}$ & 9 & 8 & 3 & 5 & 0 & 0 & 0 & 0 \\
\hline $\mathrm{Jan}$ & 4 & 2 & 0 & 3 & 1 & 0 & 0 & 0 \\
\hline $\mathrm{Fb}$ & 2 & 0 & 0 & 1 & 0 & 0 & 0 & 0 \\
\hline $\mathrm{Mr}$ & 2 & 0 & 0 & 5 & 0 & 0 & 0 & 0 \\
\hline $\mathrm{Ap}$ & 0 & 0 & 0 & 0 & 1 & 0 & 0 & 0 \\
\hline $\mathrm{My}$ & 1 & 0 & 1 & 0 & 4 & 17 & 6 & 15 \\
\hline $\mathrm{Jn}$ & 0 & 0 & 1 & 0 & 6 & 30 & 35 & 7 \\
\hline $\mathrm{Jl}$ & 0 & 0 & 0 & 0 & 45 & 40 & 62 & 22 \\
\hline $\mathrm{Ag}$ & 18 & 28 & 23 & 7 & 14 & 23 & 54 & 23 \\
\hline $\mathrm{St}$ & 56 & 27 & 23 & 24 & 15 & 6 & 2 & 1 \\
\hline $\mathrm{OC}$ & 74 & 49 & 74 & 54 & 0 & 2 & 0 & 0 \\
\hline $\mathrm{Nv}$ & 63 & 72 & 18 & 9 & 0 & 1 & 0 & 0 \\
\hline
\end{tabular}

that the juvenile specimens were also very parasitised in paddock C. From these results it can be inferred that molluscs are more likely to get infected if they can contact more easily with larvae from the faeces.

The maximum and average of total of larvae and of L3 (Tables IV and $V$ ) can be noticed to have varied throughout the year, finding the highest numbers from August to December for adult specimens and from May to September for juvenile ones. In every case the larvae/mollusc minimum was 1 . In the paddocks with a

TABLEAU IV. - Average number of total larvae (1,2 and 3) found in the four paddocks. The figures in brackets () refer to the maximum number of larvae per mollusc.

\begin{tabular}{|c|c|c|c|c|c|c|c|c|}
\hline \multirow{2}{*}{ MONTHS } & \multicolumn{4}{|c|}{ ADULT MOLLUSCS } & \multicolumn{4}{|c|}{ JUVENILE MOLLUSCS } \\
\hline & $\mathrm{A}$ & $\mathrm{B}$ & $\mathrm{C}$ & $\mathrm{D}$ & A & B & $\mathrm{C}$ & $\mathrm{D}$ \\
\hline Dc & $\begin{array}{l}(3) \\
2.1\end{array}$ & $\begin{array}{c}(10) \\
3.3\end{array}$ & $\begin{array}{l}(1) \\
1.0\end{array}$ & $\begin{array}{l}(7) \\
2.2\end{array}$ & 0 & 0 & 0 & 0 \\
\hline Jan & $\begin{array}{r}(7) \\
3.0 \\
\end{array}$ & $\begin{array}{l}(2) \\
1.5 \\
\end{array}$ & 0 & $\begin{array}{l}(2) \\
1.3\end{array}$ & $\begin{array}{l}(1) \\
1.0 \\
\end{array}$ & 0 & 0 & 0 \\
\hline $\mathrm{Fb}$ & $\begin{array}{l}(1) \\
1.0 \\
\end{array}$ & 0 & 0 & $\begin{array}{l}(6) \\
6.0 \\
\end{array}$ & 0 & 0 & 0 & 0 \\
\hline $\mathrm{Mr}$ & $\begin{array}{l}(2) \\
1.5 \\
\end{array}$ & 0 & 0 & $\begin{array}{l}(9) \\
3.4 \\
\end{array}$ & 0 & 0 & 0 & 0 \\
\hline Ap & 0 & 0 & 0 & 0 & $\begin{array}{l}(2) \\
2.0 \\
\end{array}$ & 0 & 0 & 0 \\
\hline My & $\begin{array}{r}(4) \\
4.0 \\
\end{array}$ & 0 & $\begin{array}{l}(5) \\
5.0 \\
\end{array}$ & 0 & $\begin{array}{l}(6) \\
2.7 \\
\end{array}$ & $\begin{array}{l}(5) \\
1.6 \\
\end{array}$ & $\begin{array}{r}(9) \\
2.5 \\
\end{array}$ & $\begin{array}{l}(2) \\
1.2 \\
\end{array}$ \\
\hline Jn & 0 & 0 & $\begin{array}{r}(2) \\
2.0 \\
\end{array}$ & 0 & $\begin{array}{l}(7) \\
2.6 \\
\end{array}$ & $\begin{array}{l}(10) \\
2.4\end{array}$ & $\begin{array}{r}(7) \\
2.2 \\
\end{array}$ & $\begin{array}{l}\text { (2) } \\
1.1\end{array}$ \\
\hline $\mathrm{J} 1$ & 0 & 0 & 0 & 0 & $\begin{array}{l}(8) \\
1.8 \\
\end{array}$ & $\begin{array}{r}(5) \\
1.5 \\
\end{array}$ & $\begin{array}{c}(14) \\
2.4 \\
\end{array}$ & $\begin{array}{l}(3) \\
1.2 \\
\end{array}$ \\
\hline $\mathrm{Ag}$ & $\begin{array}{l}(5) \\
1.3 \\
\end{array}$ & $\begin{array}{c}(17) \\
2.5\end{array}$ & $\begin{array}{l}(6) \\
2.1 \\
\end{array}$ & $\begin{array}{l}(2) \\
1.8 \\
\end{array}$ & $\begin{array}{l}(2) \\
1.2 \\
\end{array}$ & $\begin{array}{l}(2) \\
1.1 \\
\end{array}$ & $\begin{array}{l}(5) \\
1.6 \\
\end{array}$ & $\begin{array}{r}(8) \\
1.8 \\
\end{array}$ \\
\hline St & $\begin{array}{l}(7) \\
1.4 \\
\end{array}$ & $\begin{array}{c}(12) \\
2.0\end{array}$ & $\begin{array}{l}(3) \\
1.2 \\
\end{array}$ & $\begin{array}{l}\text { (3) } \\
1.1\end{array}$ & $\begin{array}{l}(7) \\
1.5 \\
\end{array}$ & $\begin{array}{l}(2) \\
1.5\end{array}$ & $\begin{array}{l}(1) \\
1.0\end{array}$ & $\begin{array}{l}(1) \\
1.0 \\
\end{array}$ \\
\hline Oc & $\begin{array}{r}(11) \\
2.8 \\
\end{array}$ & $\begin{array}{c}(13) \\
2.2 \\
\end{array}$ & $\begin{array}{l}(7) \\
1.5 \\
\end{array}$ & $\begin{array}{c}(15) \\
2.6 \\
\end{array}$ & 0 & $\begin{array}{l}(2) \\
1.5 \\
\end{array}$ & 0 & 0 \\
\hline $\mathrm{Nv}$ & $\begin{array}{l}(9) \\
2.0\end{array}$ & $\begin{array}{c}(17) \\
2.4 \\
\end{array}$ & $\begin{array}{c}(14) \\
1.7\end{array}$ & $\begin{array}{l}\text { (1) } \\
1.0\end{array}$ & 0 & $\begin{array}{l}\text { (1) } \\
1.0\end{array}$ & 0 & 0 \\
\hline
\end{tabular}

TABLEAU V. - Average number of larvae 3, showing the maximum number of $L 3$ per mollusc in brackets ().

\begin{tabular}{|c|c|c|c|c|c|c|c|c|}
\hline \multirow{2}{*}{ MONTHS } & \multicolumn{4}{|c|}{ ADULT MOLLUSCS } & \multicolumn{4}{|c|}{ JUVENILE MOLLUSCS } \\
\hline & A & B & C & D & A & B & C & D \\
\hline DC & $\begin{array}{l}\text { (3) } \\
1.7 \\
\end{array}$ & $\begin{array}{l}(5) \\
2.3 \\
\end{array}$ & $\begin{array}{l}(1) \\
1.0 \\
\end{array}$ & $\begin{array}{l}\text { (3) } \\
1.7\end{array}$ & 0 & 0 & 0 & 0 \\
\hline Jan & $\begin{array}{l}(1) \\
1.0\end{array}$ & 0 & 0 & $\begin{array}{l}(2) \\
2.0\end{array}$ & $\begin{array}{l}\text { (1) } \\
1.0\end{array}$ & 0 & 0 & 0 \\
\hline $\mathrm{Fb}$ & $\begin{array}{l}(1) \\
1.0\end{array}$ & 0 & 0 & $\begin{array}{l}\text { (3) } \\
3.0\end{array}$ & 0 & 0 & 0 & 0 \\
\hline $\mathrm{Mr}$ & $\begin{array}{l}(2) \\
1.0\end{array}$ & 0 & 0 & $\begin{array}{l}(2) \\
1.3\end{array}$ & 0 & 0 & 0 & 0 \\
\hline Ap & 0 & 0 & 0 & 0 & $\begin{array}{l}\text { (1) } \\
1.0\end{array}$ & 0 & 0 & 0 \\
\hline My & $\begin{array}{l}(1) \\
1.0 \\
\end{array}$ & 0 & $\begin{array}{l}\text { (3) } \\
3.0\end{array}$ & 0 & $\begin{array}{l}(5) \\
3.0\end{array}$ & $\begin{array}{r}(3) \\
1.3 \\
\end{array}$ & $\begin{array}{l}(1) \\
1.0\end{array}$ & $\begin{array}{r}(2) \\
1.4\end{array}$ \\
\hline $\mathrm{Jn}$ & 0 & 0 & $\begin{array}{l}(1) \\
1.0 \\
\end{array}$ & 0 & $\begin{array}{l}(5) \\
1.8 \\
\end{array}$ & $\begin{array}{l}(4) \\
1.8 \\
\end{array}$ & $\begin{array}{l}(6) \\
2.0\end{array}$ & $\begin{array}{l}(2) \\
1.5\end{array}$ \\
\hline Jl & 0 & 0 & 0 & 0 & $\begin{array}{r}(8) \\
1.6 \\
\end{array}$ & $\begin{array}{r}(4) \\
1.4 \\
\end{array}$ & $\begin{array}{c}(13) \\
2.6 \\
\end{array}$ & $\begin{array}{l}(2) \\
1.2\end{array}$ \\
\hline Ag & $\begin{array}{l}(4) \\
1.4 \\
\end{array}$ & $\begin{array}{c}(15) \\
2.2 \\
\end{array}$ & $\begin{array}{l}(5) \\
1.6 \\
\end{array}$ & $\begin{array}{l}(2) \\
1.3 \\
\end{array}$ & $\begin{array}{l}(2) \\
1.3 \\
\end{array}$ & $\begin{array}{l}(2) \\
1.3 \\
\end{array}$ & $\begin{array}{l}\text { (4) } \\
1.6\end{array}$ & $\begin{array}{r}(8) \\
2.0 \\
\end{array}$ \\
\hline St & $\begin{array}{l}(6) \\
2.5 \\
\end{array}$ & $\begin{array}{l}(7) \\
2.2 \\
\end{array}$ & $\begin{array}{l}(3) \\
1.2 \\
\end{array}$ & $\begin{array}{l}(2) \\
1.1 \\
\end{array}$ & $\begin{array}{l}(6) \\
1.7 \\
\end{array}$ & $\begin{array}{l}(2) \\
1.6 \\
\end{array}$ & $\begin{array}{l}(1) \\
1.0 \\
\end{array}$ & $\begin{array}{l}(1) \\
1.0 \\
\end{array}$ \\
\hline Oc & $\begin{array}{l}(9) \\
1.2 \\
\end{array}$ & $\begin{array}{l}(10) \\
2.0\end{array}$ & $\begin{array}{l}(7) \\
1.6\end{array}$ & $\begin{array}{c}(12) \\
2.3\end{array}$ & 0 & $\begin{array}{l}(1) \\
1.0\end{array}$ & 0 & 0 \\
\hline $\mathrm{Nv}$ & $\begin{array}{l}(9) \\
1.8\end{array}$ & $\begin{array}{c}(17) \\
2.6\end{array}$ & $\begin{array}{l}\text { (8) } \\
1.6\end{array}$ & $\begin{array}{l}\text { (1) } \\
1.0\end{array}$ & 0 & $\begin{array}{l}(1) \\
1.0\end{array}$ & 0 & 0 \\
\hline
\end{tabular}

lower grass height (A, B), the highest figures for the maximum and average total of larvae and of L3 for adult specimens were obtained, whereas these figures were higher for the juvenile specimens in paddock $C$.

When adult and juvenile specimens are compared, it is noticed that, in general, a larger number of adults, either living or dead, was collected. Moreover, they were more highly parasitised and presented higher figures in the total of larvae and L3 per mollusc than the juvenile specimens. By using the Chi squared test, it was shown that there were highly significant differences between adult and juvenile specimens in relation to the number of specimens collected alive $\left(\chi^{2}=154.9\right)$, dead $\left(\chi^{2}=87.2\right)$, parasitised $\left(\chi^{2}=41.9\right)$, total of larvae/mollusc $\left(\chi^{2}=155.5\right)$ and of L3 $\left(\chi^{2}=138.5\right)$.

\section{3 - Influence of climatic factors on molluscs infection}

Environmental factors had a greater influence on juvenile stages than on adult ones. By using linear regression, it is observed (Table VI) that the number of juvenile specimens collected alive as well as that of the parasitised ones, the total of larvae and L3, were correlated positively with the average temperatures of the minimum, maximum and average and with the days of mist, whereas a negative correlation was found as regards relative humidity, precipitation and days of rain.

The number of living adult specimens (Table VII) was correlated positively with the temperatures and the days of mist. The differences were not statistically significant 
TABLEAU VI. - Coefficient of correlation $\left(R^{2}\right)$ between the number of juvenile molluscs alive, infected, average of the total larvae and of $L 3$ and the different climatic parameters.

\begin{tabular}{|c|c|c|c|c|c|c|c|c|c|c|c|c|c|c|c|c|}
\hline \multirow[b]{3}{*}{ PLOTS } & \multicolumn{8}{|c|}{$M O L L U S C S$} & \multicolumn{8}{|c|}{ PARASITES } \\
\hline & \multicolumn{4}{|c|}{ Alive } & \multicolumn{4}{|c|}{ Infected } & \multicolumn{4}{|c|}{$\bar{x}$ Total larvae } & \multicolumn{4}{|c|}{$\bar{x}\llcorner 3$} \\
\hline & A & B & $c$ & D & A & $\mathrm{B}$ & $c$ & D & $\mathrm{A}$ & B & $\mathrm{C}$ & $D$ & A & $\mathrm{B}$ & $c$ & D \\
\hline Min. Temp. & $\begin{array}{c}0.72 \\
+\quad * \\
\end{array}$ & $\begin{array}{c}0.79 \\
+\quad * \\
\end{array}$ & $\begin{array}{c}0.72 \\
+\quad * \\
\end{array}$ & $\begin{array}{c}0.67 \\
+\quad * \\
\end{array}$ & $\begin{array}{c}0.61 \\
+* \\
\end{array}$ & $\begin{array}{c}0.17 \\
+ \\
\end{array}$ & $\begin{array}{r}0.69 \\
+\quad * \\
\end{array}$ & $\begin{array}{c}0.71 \\
+* \\
\end{array}$ & $\begin{array}{r}0.50 \\
+\quad * \\
\end{array}$ & $\begin{array}{r}0.86 \\
+\quad \text { } \\
\end{array}$ & $\begin{array}{r}0.76 \\
+\quad * \\
\end{array}$ & $\begin{array}{r}0.80 \\
+\quad * \\
\end{array}$ & $\begin{array}{r}0.55 \\
+\quad * \\
\end{array}$ & $\begin{array}{c}0.92 \\
+\quad * \\
\end{array}$ & $\begin{array}{c}0.78 \\
+\quad \star \\
\end{array}$ & $\begin{array}{r}0.80 \\
+\quad \\
\end{array}$ \\
\hline Max.Temp. & $\begin{array}{c}0.81 \\
+\quad * \\
\end{array}$ & $\begin{array}{c}0.86 \\
+* \\
\end{array}$ & $\begin{array}{c}0.79 \\
+\quad * \\
\end{array}$ & $\begin{array}{c}0.73 \\
+\quad * \\
\end{array}$ & $\begin{array}{c}0.70 \\
+\quad * \\
\end{array}$ & $\begin{array}{c}0.11 \\
+ \\
\end{array}$ & $\begin{array}{c}0.74 \\
+\quad * \\
\end{array}$ & $\begin{array}{c}0.77 \\
+\quad * \\
\end{array}$ & $\begin{array}{c}0.54 \\
+\quad \star \\
\end{array}$ & $\begin{array}{c}0.80 \\
+\quad * \\
\end{array}$ & $\begin{array}{c}0.82 \\
+\quad * \\
\end{array}$ & $\begin{array}{c}0.89 \\
+\quad * \\
\end{array}$ & $\begin{array}{c}0.64 \\
+* \\
\end{array}$ & $\begin{array}{c}0.85 \\
+\quad * \\
\end{array}$ & $\begin{array}{r}0.85 \\
+\quad * \\
\end{array}$ & $\begin{array}{c}0.86 \\
+* \\
\end{array}$ \\
\hline Aver. Temp. & $\begin{array}{c}0.78 \\
+\quad * \\
\end{array}$ & $\begin{array}{c}0.85 \\
+* \\
\end{array}$ & $\begin{array}{c}0.78 \\
+* \\
\end{array}$ & $\begin{array}{c}0.72 \\
+* \\
\end{array}$ & $\begin{array}{l}0.67 \\
+*\end{array}$ & $\begin{array}{c}0.15 \\
+\end{array}$ & $\begin{array}{c}0.73 \\
+\quad * \\
\end{array}$ & $\begin{array}{c}0.76 \\
+\quad * \\
\end{array}$ & $\begin{array}{c}0.54 \\
+\quad * \\
\end{array}$ & $\begin{array}{c}0.85 \\
+\quad * \\
\end{array}$ & $\begin{array}{c}0.81 \\
+\quad \text { * } \\
\end{array}$ & $\begin{array}{r}0.87 \\
+* \\
\end{array}$ & $\begin{array}{c}0.61 \\
+* \\
\end{array}$ & $\begin{array}{c}0.91 \\
+\quad * \\
\end{array}$ & $\begin{array}{c}0.84 \\
+\quad * \\
\end{array}$ & $\begin{array}{c}0.85 \\
+\quad \text { * } \\
\end{array}$ \\
\hline Frost days & 0.48 & 0.49 & 0.45 & 0.41 & 0.39 & 0.48 & 0.42 & 0.45 & $\underset{\star}{0.55}$ & 0.40 & $\underset{*}{0.55}$ & $\underset{\star}{0.57}$ & $\underset{*}{0.54}$ & $\underset{*}{0.50}$ & $\underset{*}{0.53}$ & $\underset{*}{0.57}$ \\
\hline Rel.humid. & 0.37 & $\underset{*}{0.53}$ & $\underset{*}{0.55}$ & $\underset{*}{0.55}$ & 0.26 & 0.40 & $\underset{*}{0.54}$ & $\underset{*}{0.53}$ & $\underset{*}{0.71}$ & 0.27 & $\underset{*}{0.60}$ & $\underset{*}{0.61}$ & $\underset{\star}{0.65}$ & 0.26 & $\underset{*}{0.57}$ & $\underset{*}{0.65}$ \\
\hline Precipit. & $\underset{\star}{0.56}$ & $\underset{\star}{0.56}$ & 0.50 & 0.47 & $\underset{*}{0.51}$ & 0.04 & 0.50 & 0.48 & 0.40 & 0.41 & $\underset{*}{0.57}$ & 0.62 & $\underset{*}{0.52}$ & $\underset{*}{0.50}$ & $\underset{*}{0.62}$ & $\underset{*}{0.61}$ \\
\hline Rain days & $\underset{\star}{0.61}$ & $\underset{*}{0.57}$ & $\underset{\star}{0.52}$ & 0.49 & $\begin{array}{c}0.55 \\
+\quad * \\
\end{array}$ & 0.37 & $\underset{\star}{0.52}$ & 0.49 & 0.17 & 0.38 & $\underset{*}{0.50}$ & $\underset{*}{0.60}$ & 0.35 & 0.44 & $\underset{*}{0.58}$ & $\underset{*}{0.56}$ \\
\hline Fog days & $\begin{array}{c}0.92 \\
+*\end{array}$ & $\begin{array}{c}0.89 \\
+*\end{array}$ & $\begin{array}{c}0.82 \\
+*\end{array}$ & $\begin{array}{c}0.74 \\
+*\end{array}$ & $\begin{array}{l}0.86 \\
+*\end{array}$ & $\begin{array}{c}0.05 \\
+\end{array}$ & $\begin{array}{l}0.80 \\
+\quad *\end{array}$ & $\begin{array}{l}0.77 \\
+\quad *\end{array}$ & $\begin{array}{c}0.39 \\
+\end{array}$ & $\begin{array}{c}0.55 \\
+\quad * \\
\end{array}$ & $\begin{array}{c}0.72 \\
+\quad \text { * } \\
\end{array}$ & $\begin{array}{c}0.82 \\
+\quad * \\
\end{array}$ & $\begin{array}{c}0.50 \\
+\quad * \\
\end{array}$ & $\begin{array}{c}0.66 \\
+\quad * \\
\end{array}$ & $\begin{array}{c}0.85 \\
+\quad \text { * } \\
\end{array}$ & $\begin{array}{c}0.76 \\
+\quad * \\
\end{array}$ \\
\hline
\end{tabular}

$+=$ positive relation

* = significant differences

TABLEAU VII. - Coefficient of correlation $\left(R^{2}\right)$ between the number of adult molluscs found alive, infected, average of the total larvae and of $L 3$ and the different climatic parameters.

\begin{tabular}{|c|c|c|c|c|c|c|c|c|c|c|c|c|c|c|c|c|}
\hline \multirow[b]{3}{*}{ PLOTS } & \multicolumn{8}{|c|}{$M O L L U S C S$} & \multicolumn{8}{|c|}{ PARASITES } \\
\hline & \multicolumn{4}{|c|}{ Alive } & \multicolumn{4}{|c|}{ Infected } & \multicolumn{4}{|c|}{$\bar{x}$ Total larvae } & \multicolumn{4}{|c|}{$\bar{x}\llcorner 3$} \\
\hline & A & B & $c$ & $D$ & A & B & C & $D$ & A & $\mathrm{B}$ & C & $D$ & A & B & C & $D$ \\
\hline Min. Temp. & $\begin{array}{c}0.43 \\
+\end{array}$ & $\begin{array}{c}0.53 \\
+*\end{array}$ & $\begin{array}{c}0.54 \\
+*\end{array}$ & $\begin{array}{c}0.54 \\
+\quad \text { * }\end{array}$ & $\begin{array}{c}0.28 \\
+\end{array}$ & $\begin{array}{c}0.30 \\
+\end{array}$ & $\begin{array}{c}0.22 \\
+\end{array}$ & $\begin{array}{c}0.02 \\
+\end{array}$ & $\begin{array}{c}0.20 \\
+\end{array}$ & $\begin{array}{c}0.06 \\
+\end{array}$ & $\begin{array}{c}0.49 \\
+\end{array}$ & $\begin{array}{l}0.50 \\
+\quad * \\
\end{array}$ & $\begin{array}{c}0.11 \\
+\end{array}$ & $\begin{array}{c}0.01 \\
+ \\
\end{array}$ & $\begin{array}{r}0.50 \\
+\quad * \\
\end{array}$ & $\begin{array}{c}0.55 \\
+\quad * \\
\end{array}$ \\
\hline Max.Temp. & $\begin{array}{c}0.50 \\
+*\end{array}$ & $\begin{array}{c}0.60 \\
+*\end{array}$ & $\begin{array}{l}0.60 \\
+*\end{array}$ & $\begin{array}{c}0.61 \\
+\star\end{array}$ & $\begin{array}{c}0.12 \\
+\end{array}$ & $\begin{array}{c}0.03 \\
+\end{array}$ & $\begin{array}{c}0.12 \\
+\end{array}$ & 0.32 & $\begin{array}{c}0.14 \\
+\end{array}$ & $\begin{array}{c}0.02 \\
+\end{array}$ & $\begin{array}{c}0.44 \\
+\end{array}$ & $\begin{array}{c}0.41 \\
+ \\
\end{array}$ & $\begin{array}{c}0.10 \\
+\end{array}$ & $\begin{array}{c}0.13 \\
+ \\
\end{array}$ & $\begin{array}{c}0.43 \\
+ \\
\end{array}$ & 0.13 \\
\hline Aver. Temp. & $\begin{array}{c}0.48 \\
+\end{array}$ & $\begin{array}{l}0.58 \\
+*\end{array}$ & $\begin{array}{c}0.58 \\
+*\end{array}$ & $\begin{array}{c}0.59 \\
+*\end{array}$ & $\begin{array}{c}0.20 \\
+\end{array}$ & $\begin{array}{c}0.16 \\
+\end{array}$ & $\begin{array}{c}0.05 \\
+\end{array}$ & $\begin{array}{c}0.16 \\
+\end{array}$ & $\underset{+}{0.18}$ & $\begin{array}{c}0.04 \\
+\end{array}$ & $\begin{array}{c}0.48 \\
+\end{array}$ & $\begin{array}{c}0.45 \\
+\end{array}$ & $\begin{array}{c}0.10 \\
+\end{array}$ & $\begin{array}{c}0.17 \\
+\end{array}$ & $\begin{array}{c}0.51 \\
+*\end{array}$ & $\begin{array}{r}0.50 \\
+*\end{array}$ \\
\hline Frost days & 0.20 & 0.07 & 0.01 & 0.01 & $\begin{array}{c}0.17 \\
+\end{array}$ & $\begin{array}{c}0.07 \\
+\end{array}$ & 0.12 & $\begin{array}{c}0.80 \\
+\end{array}$ & 0.44 & $\begin{array}{c}0.53 \\
+*\end{array}$ & 0.22 & $\begin{array}{c}0.37 \\
+\end{array}$ & $\begin{array}{c}0.25 \\
+\end{array}$ & $\begin{array}{c}0.26 \\
+\end{array}$ & 0.10 & $\begin{array}{c}0.65 \\
+\quad \text { * } \\
\end{array}$ \\
\hline Rel.humid. & $\begin{array}{c}0.13 \\
+\end{array}$ & $\begin{array}{c}0.16 \\
+\end{array}$ & $\begin{array}{c}0.12 \\
+\end{array}$ & $\begin{array}{c}0.01 \\
+\end{array}$ & $\begin{array}{c}0.60 \\
+*\end{array}$ & $\begin{array}{c}0.57 \\
+*\end{array}$ & $\begin{array}{c}0.62 \\
+*\end{array}$ & $\begin{array}{c}0.75 \\
+*\end{array}$ & $\begin{array}{c}0.14 \\
+\end{array}$ & $\begin{array}{c}0.16 \\
+\end{array}$ & 0.24 & $\begin{array}{c}0.53 \\
+*\end{array}$ & $\begin{array}{c}0.45 \\
+\end{array}$ & $\begin{array}{c}0.33 \\
+\end{array}$ & 0.06 & $\begin{array}{c}0.50 \\
+\end{array}$ \\
\hline Precipit. & 0.16 & 0.26 & 0.25 & 0.32 & $\begin{array}{c}0.15 \\
+\end{array}$ & $\begin{array}{c}0.25 \\
+\end{array}$ & $\begin{array}{c}0.33 \\
+\end{array}$ & $\begin{array}{c}0.54 \\
+\end{array}$ & 0.17 & 0.20 & 0.20 & $\begin{array}{c}0.45 \\
+\end{array}$ & 0.10 & 0.08 & 0.15 & $\begin{array}{c}0.32 \\
+\end{array}$ \\
\hline Rain days & 0.39 & 0.47 & 0.44 & 0.49 & $\begin{array}{c}0.38 \\
+\end{array}$ & $\begin{array}{c}0.20 \\
+\end{array}$ & $\begin{array}{c}0.38 \\
+\end{array}$ & $\begin{array}{c}0.49 \\
+\end{array}$ & 0.04 & 0.18 & 0.09 & 0.04 & 0.16 & 0.01 & 0.09 & 0.17 \\
\hline Fog days & $\begin{array}{c}0.38 \\
+\end{array}$ & $\begin{array}{r}0.54 \\
+*\end{array}$ & $\begin{array}{l}0.52 \\
+\quad *\end{array}$ & $\begin{array}{l}0.56 \\
+*\end{array}$ & 0.04 & 0.13 & 0.28 & 0.45 & 0.31 & 0.10 & 0.11 & 0.25 & 0.11 & 0.02 & $\begin{array}{c}0.09 \\
+\end{array}$ & 0.33 \\
\hline
\end{tabular}

$+=$ positive relation

* = signifficant differences

with the rest of the climatic factors. In general, it was observed that the number of parasitised adults and the total of larvae and of L3 were correlated positively with the temperature and the relative humidity, but the differences, in general, were not significant.
The species of Protostrongylidae identified in the molluscs (adult and juvenile) correspond with the L1 found in the faeces from the sheep that grazed in the mentioned paddocks. The frequency and intensity, listed from highest to lowest was: $N$. linearis, $M$. capillaris, and 


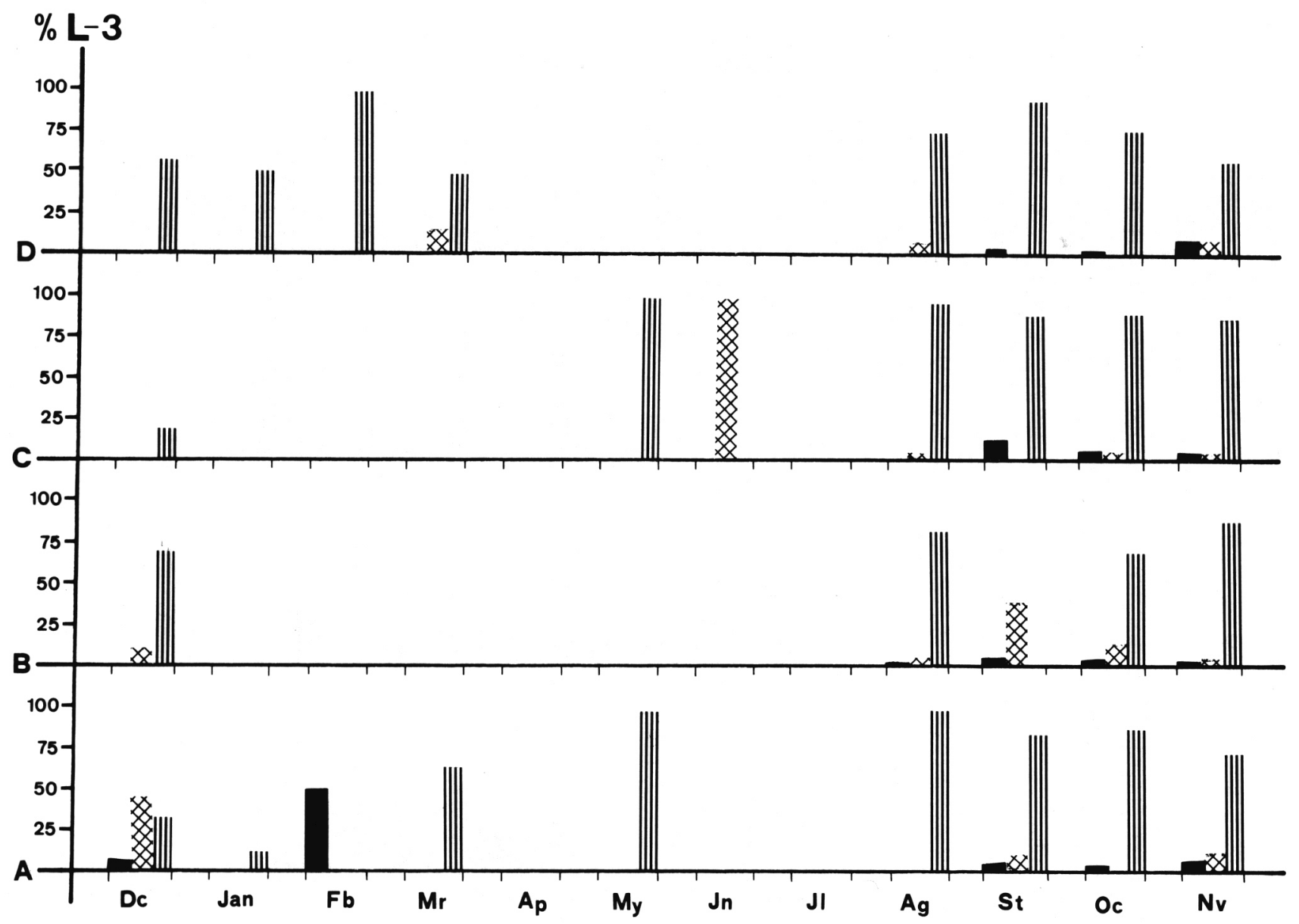

FIG. 2. - Percentages of larvae 3 of Cystocaulus ocreatus ( $\bullet$ ), Muellerius capillaris $(\mathbf{X})$ and Neostrongylus linearis (IIII) related to the total number of L3 found in the adult molluscs in paddocks A, B, C and D from December 1988 to Novembrer 1989.

C. ocreatus. In the different paddocks, in adult snails (Fig. 2), the highest percentage of L3 was found mainly at the end of summer and during autumn. In the coldest months (December-March) the prevalence of L3 happened in paddocks $\mathrm{A}$ and $\mathrm{D}$. In these paddocks the most frequent was $N$. linearis, followed in a lower proportion by $M$. capillaris, and $C$. ocreatus was only found in the adult specimens from paddock A.

In juvenile specimens (Fig. 3) the Protostrongylidae species frequency order was the same as the adults. In every paddock the highest percentages of $N$. linearis, $M$. capillaris and $C$. ocreatus were noticed from May to September. The L3 stage of these nematodes were only observed in january and April in paddock $\mathrm{A}$ and in October and November in paddock $B$.

\section{DISCUSSION}

Living $C$. barbara were collected throughout the period of study, with a proportion of adults which agrees with what was found by Manga (1983) in the province of León (Spain). Adult molluscs prevailed in autumn-winter and juvenile ones in spring-summer, which agrees with what was observed in Morocco by Cabaret et al. (1980) and Cabaret (1988).

Dead snails were found throughout the year, but a higher mortality rate was recorded among the juvenile specimens in summer, when rainfall was scarce, which agrees with what was obtained by Cabaret (1986).

The number of parasitised adult specimens as well as the prevalence and intensity of infection by Protostrongylidae larvae was higher in adult specimens than in juvenile ones, which agrees with what was observed by Cabaret et al. (1980) and Cabaret $(1986,1988)$. The maximum number and the average of the total of larvae and L3 were lower in this experiment when compared to those found by us (Morrondo and Manga, 1982; Morrondo et al., 1982 and Díez et al., 1987) when Cochlicella barbara was infected experimentally with larvae of $C$. ocreatus, $M$. capillaris and $N$. linearis. Likewise, the maximum and average numbers of the total of larvae and L3 of $N$. linearis and 


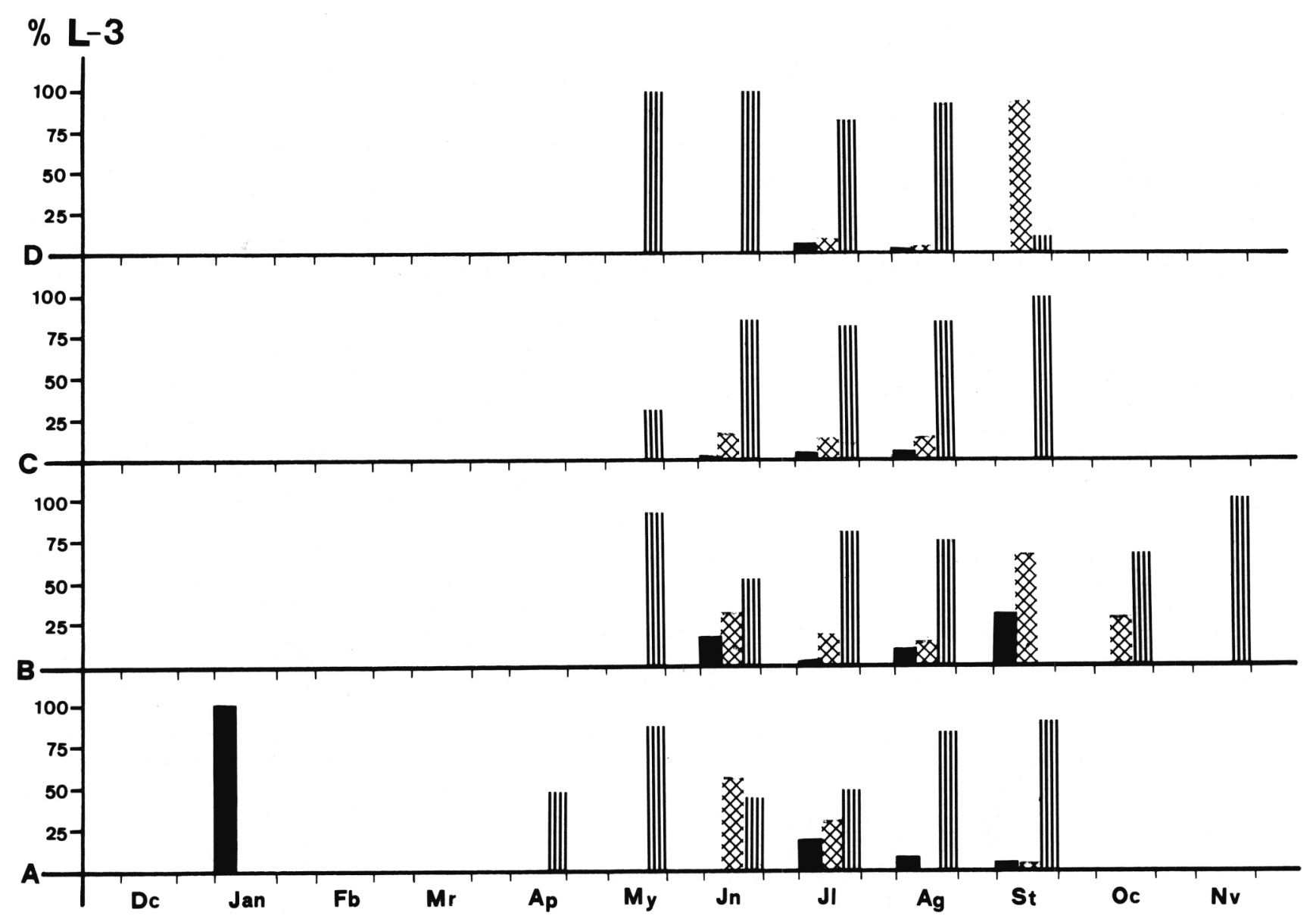

FIG. 3. - Percentages of larvae 3 of Cystocaulus ocreatus ( $\square$ ), Muellerius capillaris $(\boldsymbol{X} \times$ ) and Neostrongylus linearis (IIII) related to the total number of L3 found in the juvenile molluscs in paddocks A, B, C and D from December 1988 to November 1989.

M. capillaris were lower than the ones found by us (Morrondo et al., 1987, 1988) when they were infected in the laboratory Cernuella (Xeromagna) cespitum arigonis and they were taken later to natural conditions. On the contrary, in this study the maximum and average figures of the total of larvae and L3 were higher than those found by Cabaret et al. (1980) and Cabaret (1981, 1986, 1988) in experiments carried out with this and other species of Cochlicella.

It was noticed that the different climatic factors have a greater influence on juvenile specimens than on adult ones. In both cases, there was a positive correlation with temperature and a negative one with monthly average rainfall, which agrees with what was observed by Cabaret (1988). The behaviour of the juvenile molluscs had a positive correlation with temperature and a negative one with respect to relative humidity, which agrees with what was observed by us when $C$. (Xeromagna) cespitum arigonis was infected in the laboratory and kept in natural environment (Morrondo et al., 1987, 1988).

The maximum and average figures of L3 were found from mid spring to mid autumn, so that this period may be considered as the one with a greater risk for the infection of the definitive hosts, which agrees with what was found (Morrondo et al., 1987, 1988) in the molluscs kept in natural conditions and, in general, with what was observed by Cabaret et al. (1980), Cabaret (1981, 1986, 1988) and Dakkak and Cabaret (1984) in Morocco, where they showed that the periods with a greater ovine infection risk are last autumn and early winter, in spite of the lower and later rainfall registered in our area.

From our results it is inferred that the definitive hosts are more likely to get infected from mid spring to mid autumn, above all during the summer, because there are more molluscs with third larvae at the disposal of the ovine hosts in the paddocks. Likewise, the exploitation of paddocks with a low grass height (that is to say, with a higher number of sheep grazing, which means that the paddocks receive more infective for the molluscs first stage larvae) will imply a greater posibility of ovine infection. Considering the period of prevalence of Protostrongylidae, the results agree with what was stated by Martínez et al. (1989) 
in relation to the higher elimination of L1, in winter, by the sheep that grazed in the paddocks studied.

Acknowledgments. - We are grateful to Dra. Y. MANGAGonZalez, researcher in the Consejo Superior de Investigaciones Científicas (León, Spain) for identification of the molluscs. To Dr. J. CABARET, researcher in the Institut National de la Recherche Agronomique (Nouzilly, France) for advising us on the statistical methods that we have used in this study.

\section{REFERENCES}

Cabaret J. : Utilisation de Cochlicella conoidea comme indicateur du parasitisme à Protostrongylidés sur les prairies. Haliotis, 1981, $11,73-77$.

Cabaret J. : Survie des larves de protostrongles dans les fèces et infestation dirigée des mollusques, à Rabat (Maroc). Rev. Elev. Med. Vet. Pays trop., 1986, 39, 323-331.

Cabaret J. : Natural infection of land-snails by Protostrongylids on a pasture grazed by sheep in the Rabat area of Morocco. Vet. Parasitol., 1988, 26, 297-304.

Cabaret J., Dakkak A., Bahaida B. : Étude de l'infestation des mollusques terrestres de la région de Rabat (Maroc) par les larves de protostrongylidés dans les conditions naturelles. Rev. Elev. Med. Vet. Pays trop., 1980, 33, 159-165.

Dakkak A., Cabaret J. : Des Mollusques terrestres hôtes intermédiaires de Protostrongylides dans les pâturages de la région de Rabat. Actes Inst. Agro Vet., 1984, 4, 41-46.
Díez-Baños P., Díez-Baños N., Núñez-Gutiérrez M. C., MorrondoPelayo M. P. : Infestación experimental de Cochlicella barbara (Mollusca, Helicidae) con larvas de Muellerius sp. y Neostrongylus linearis (Nematoda, Protostrongylidae) procedentes de rebeco. Rev. Iber. Parasitol., 1987, Vol. Extraordinario, 179-187.

Manga M. Y. : Los Helicidae (Gastropoda, Pulmonata) de la provincia de Leon. Institución Fray Bernardino de Sahagún. Excelentisima Diputación Provincial de León, Consejo Superior de Investigaciones Cientificas (CECEL), 1983, 1-394.

Manga M. Y., Morrondo M. P., Cordero M. : Moluscos hospedadores intermediarios de Protostrongylidae ovinos. Universidad de León, León, 1986, 1-136.

Martínez-Nistal M. C., Díez-Baños P., Mezo-Menéndez M. : Ritmos de eliminación larvaria de helmintos pulmonares en ovinos gallegos. VI Congreso Nacional y I Congreso Ibérico de Parasitología. Cáceres (Spain), 1989, 219.

Morrondo-Pelayo M. P., Manga-González M. Y. : Experimental study on the susceptibility of five Helicidae species to larvae of Protostrongylinae. Malacologia, 1982, 22, 23-28.

Morrondo-Pelayo M.P., Manga-González M. Y., Cordero-delCampillo M., Díez-Baños P., Díez-Baños N. : Development of Neostrongylus linearis (Nematoda, Protostrongylidae) larvae in Cernuella cespitum arigonis (Mollusca, Stylommatophora) infected in the laboratory and kept in its natural environment. Angew. Parasitol., 1987, 28, 37-45.

Morrondo-Pelayo M. P., Manga-González M. Y., Cordero-delCampillo M., Díez-Baños P., Díez-Baños N. : Larval development of Muellerius capillaris (Nematoda, Protostrongylidae) in experimentally infected Cernuella (Xeromagna) cespitum arigonis (Mollusca, Helicidae). J. Moll. Stud., 1988, 54, 21-34. 